\title{
Mutants of Neurospora crassa, Escherichia coli and Salmonella typhimurium Specifically Inhibited by Carbon Dioxide
}

\author{
By GILLIAN A. ROBERTS AND H. P. CHARLES \\ Department of Microbiology, The University, London Road, Reading, RGI $5 A Q$ \\ (Accepted for publication I June 1970) \\ SUMMAR Y \\ Mutants of Neurospora crassa, Escherichia coli and Salmonella typhimurium \\ are described which are inhibited by $\mathrm{CO}_{2}$ at concentrations which do not \\ inhibit the parental strains from which the mutants were derived. Sensitivity \\ to inhibition by $\mathrm{CO}_{2}$ is caused by single gene mutations. The $\mathrm{CO}_{2}$ inhibitions \\ are reversed by specific substances; for example, the $\mathrm{CO}_{2}$ inhibition of a \\ methionine-requiring mutant of $N$. crassa is reversed by purines, and the $\mathrm{CO}_{2}$ \\ inhibition of a prototroph of $E$. coli is reversed by methionine or vitamin $\mathrm{B}_{12}$. \\ The nature of the defects in the $\mathrm{CO}_{2}$-inhibited mutants is discussed.
}

\section{INTRODUCTION}

Several mutants of Neurospora crassa and Escherichia coli grow in minimal medium without their specific growth factors when the gas phase is air containing 20 to $30 \%$ ( $\mathrm{v} / \mathrm{v})$ of carbon dioxide (Broadbent \& Charles, 1965; Charles \& Roberts, 1968). Mutants which are stimulated by $\mathrm{CO}_{2}$ in this way are called ' $\mathrm{CO}_{2}$ mutants' (Charles \& Broadbent, 1964); they are not usually stimulated by bicarbonate. The mechanism of the stimulation is not known with certainty and may not be the same for all $\mathrm{CO}_{2}$ mutants. In some $\mathrm{CO}_{2}$ mutants the mutations affect enzymes which catalyse $\mathrm{CO}_{2}-$ incorporation reactions, and it is probable that high concentrations of $\mathrm{CO}_{2}$ may compensate for decreased enzymic activity by a mass action effect; this may be the mechanism of the stimulation in some purine-requiring and arginine-requiring mutants which are $\mathrm{CO}_{2}$ mutants. In other $\mathrm{CO}_{2}$ mutants, for example certain mutants of $E$. coli which require isoleucine + valine, the mutations apparently affect enzymes which do not catalyse $\mathrm{CO}_{2}$-incorporation reactions and it is not known how $\mathrm{CO}_{2}$ stimulates their growth.

$\mathrm{CO}_{2}$ mutants have also recently been isolated from Salmonella typhimurium (H. P. Charles, unpublished).

The present report describes mutants of a new class; these are inhibited rather than stimulated by $\mathrm{CO}_{2}$. These mutants will be called ' $\mathrm{CO}_{2}$-inhibited mutants'. They have been found in Neurospora crassa, Escherichia coli and Salmonella typhimurium. They are inhibited by concentrations of $\mathrm{CO}_{2}$ which stimulate the $\mathrm{CO}_{2}$-requiring mutants and which do not inhibit the parental strains from which the $\mathrm{CO}_{2}$-inhibited mutants are derived. 


\section{METHODS}

Strains. The Neurospora crassa wild-type strains used were EMERSON $5256 \mathrm{~A}$ and EMERSON 5297a, kindly provided by Professor D. G. Catcheside (Department of Genetics, John Curtin School of Medical Research, Australian National University, Canberra, A.C.T., Australia), and 74-OR 23-I $A$ and 74-OR 8-I $a$, kindly provided by Dr F. J. de Serres (Oak Ridge National Laboratory, U.S.A.). The $N$. crassa methioninerequiring strains met-I (35599), met-2 (K44), met-3 (36104), met-4 (39816), met-5 (9666), met-6 (35809), met-7 (4894), met-8 (P 53) and met-9 (Cr 24), the lysine-requiring strains lys-2 (37IOI $a$ ) and lys-3 (288I5) and the para-aminobenzoic acid-requiring strain pab-I (I633A) were kindly provided by Mr W. Ogata (Fungal Genetics Stock Center, Dartmouth College, Hanover, New Hampshire 03755, U.S.A.). The methioninerequiring strains mac $(65 \mathrm{I} 08)$ and $m e$ ( $(\mathrm{s} 2706)$ were kindly provided by Dr Noreen E. Murray (M.R.C. Molecular Genetics Unit, Department of Molecular Biology, King's Buildings, West Mains Road, Edinburgh 9).

The strain of Escherichia coli from which the $\mathrm{CO}_{2}$-inhibited mutants were isolated was strain AB I62 I (F- $; 6-r$, thi, $m t l, x y l, s t r-r$, ara, gal, lac, $T 4-r)$, kindly provided by Professor E. A. Adelberg (Department of Microbiology, Yale University, New Haven, Connecticut 065Io, U.S.A.).

The $\mathrm{CO}_{2}$-inhibited mutant of Salmonella typhimurium was isolated from strain LT2, kindly provided by Dr P. E. Hartman (Johns Hopkins University, Baltimore, Maryland, U.S.A.).

Media. The minimal medium used for Neurospora crassa was that of Vogel (I956) solidified with agar (Difco Bacto, $\mathrm{I} \cdot 2 \% \mathrm{w} / \mathrm{v}$ ) when necessary. Sorbose $(5 \mathrm{~g} . / 1$.) was added to the solid minimal medium to restrict the growth of colonies. The complete medium used for N. crassa was the conidiation medium of Horowitz (Ryan, 1950).

The minimal medium used for Escherichia coli and Salmonella typhimurium was that of Vogel \& Bonner (1956). It was supplemented with thiamine (2 mg./1.) for $E$. coli, and was solidified when necessary with Difco Bacto agar $(\mathrm{I} \cdot 2 \% \mathrm{w} / \mathrm{v})$. The complete medium used contained the following (g./1.): tryptone (Oxoid), Io; yeast extract (Difco), 5; $\mathrm{KH}_{2} \mathrm{PO}_{4}, 3$; glucose, 5; agar (when required), 15.

Unless otherwise stated, all cultures of Neurospora crassa were incubated at $30^{\circ}$ and all cultures of Escherichia coli and Salmonella typhimurium at $37^{\circ}$.

The effect of $\mathrm{CO}_{2}$ on growth was tested by incubating cultures in Petri dishes in 5 litre vacuum desiccators containing an appropriate mixture of air and $\mathrm{CO}_{2}$ as described by Charles (1964).

Isolation of mutants. The $\mathrm{CO}_{2}$-inhibited mutant of Neurospora crassa, $\mathrm{F} 4$, was obtained from ultraviolet-irradiated conidia of the wild-type strain 74-OR 23-I $A$ by the filtration enrichment procedure (Catcheside, I954; Woodward, De Zeeuw \& Srb, 1954). The filtration procedure was modified in that the irradiated conidia were incubated throughout the filtration period in a gas phase of air supplemented with $\mathrm{CO}_{2}$ (about $30 \%, v / v$ ). This modification was introduced in order to suppress growth of any $\mathrm{CO}_{2}$-inhibited mutants which might be present in the population and which would otherwise grow and be removed from the suspension by filtration. The duration of the incubation period in the filtration enrichment experiment was $48 \mathrm{~h}$. and the suspension was filtered approximately every $5 \mathrm{~h}$. during this period. Samples of the final filtrate were plated on conidiation medium, and after incubation for 3 days conidia from the 
colonies which appeared were tested for ability to grow on minimal medium in air and in air supplemented with $30 \%(\mathrm{v} / \mathrm{v}) \mathrm{CO}_{2}$. The growth factor requirements of auxotrophs were determined by the auxanographic method (Pontecorvo, 1949). All auxotrophs obtained were tested to determine whether or not the response to their respective growth factor was inhibited by the presence of $30 \% \mathrm{CO}_{2}(\mathrm{v} / \mathrm{v})$ in the gas phase.

The $\mathrm{CO}_{2}$-inhibited mutants of Escherichia coli and Salmonella typhimurium were isolated as follows. Samples of an ultraviolet-irradiated cell suspension were plated on complete medium and incubated in air. Colonies which grew were then printed by the replica plating technique (Lederberg \& Lederberg, 1952) on to each of two plates of minimal medium, one of which was incubated in air and the other in air supplemented with $20 \%(\mathrm{v} / \mathrm{v}) \mathrm{CO}_{2}$. Plates were observed after incubation for $24 \mathrm{~h}$. and any colonies which failed to appear on the plates incubated in $\mathrm{CO}_{2}$-supplemented air were picked from the corresponding air-incubated plates and retested to confirm their $\mathrm{CO}_{2}$-inhibited phenotype.

Crosses. Crosses between strains of Neurospora crassa were made on cornmeal agar medium (Smith, 1954) supplemented with the growth factor requirement of the protoperithecial parent. They were incubated at $25^{\circ}$ and analysed about 2 weeks after ejection of the ascospores. Germination of the ascospores was induced by placing them at $60^{\circ}$ for $45 \mathrm{~min}$. Germinated ascospores were individually transferred to tubes of conidiation medium and the resulting cultures were tested for phenotype after incubation for 3 to 4 days.

\section{RESULTS}

One mutant of Neurospora crassa which showed inhibition by $\mathrm{CO}_{2}(30 \%, \mathrm{v} / \mathrm{v}$, in air) was a newly isolated lysine-requiring mutant, F4, derived from wild-type strain 74-OR 23-I $A$ by the procedure described in Methods. This mutant shows the characteristics of $\mathrm{CO}_{2}$-inhibited mutants in their simplest form. The sensitivity of the mutant to inhibition by $\mathrm{CO}_{2}$ was not due to the mutation which causes the lysine requirement, because a cross of the mutant to wild-type (strain 74-OR 8-I $a$ ) yielded some segregants which were inhibited by $\mathrm{CO}_{2}(30 \%, \mathrm{v} / \mathrm{v})$ but which did not require lysine. These $\mathrm{CO}_{2}$-inhibited segregants were prototrophic in ordinary air. Crosses of $\mathrm{CO}_{2}$-inhibited prototrophic segregants to wild-type strains gave only wild-type and $\mathrm{CO}_{2}$-inhibited prototrophic segregants in equal proportions, showing that $\mathrm{CO}_{2}$-sensitivity was determined by a single gene mutation. This suggested that an enzyme in the mutant did not function effectively in the presence of $30 \%(\mathrm{v} / \mathrm{v}) \mathrm{CO}_{2}$. The function of this enzyme might be revealed if the $\mathrm{CO}_{2}$-inhibition could be reversed by supplying a nutrient in the medium. By auxanographic experiments it was found that cytidine reversed the $\mathrm{CO}_{2}$-inhibition of mutant $\mathrm{F} 4$ and of the prototrophic segregants derived from it and caused them to grow as well as wild-type in the presence of $30 \%(\mathrm{v} / \mathrm{v}) \mathrm{CO}_{2}$. Other metabolites, including other pyrimidines and pyrimidine derivatives, did not reverse the $\mathrm{CO}_{2}$ inhibition. Later experiments showed that only one of several samples of cytidine reversed the $\mathrm{CO}_{2}$ inhibition, indicating that an impurity in the active sample was responsible for reversal of the inhibition. The sample of cytidine was too small to permit identification of its active component and it was not possible to obtain more cytidine of the same batch. The possibility that the impurity was a vitamin was unlikely since all available vitamins were tested on the mutant and failed to reverse the inhibition. 
Analysis of crosses between $\mathrm{CO}_{2}$-inhibited prototrophic segregants derived from mutant $\mathrm{F} 4$ and various marker strains indicated that the mutation causing sensitivity to $\mathrm{CO}_{2}$ was located in the right arm of linkage group V. Crosses with the Group VR markers lys-2 (3710I $a$ ) and pab-I (I633 $A$ ) gave recombination frequencies of 23 and $7 \%$ respectively. Crosses of $\mathrm{CO}_{2}$-inhibited prototrophs with mutants in other linkage groups gave recombination frequencies ranging from 45 to $70 \%$ recombination.

The first $\mathrm{CO}_{2}$ inhibition to be discovered was in Neurospora crassa mutant 35809 a which has a mutation at the me-6 locus (Beadle \& Tatum, I945; Perkins, I959). Under ordinary cultural conditions, when the gas phase is air, the mutant requires methionine for growth and does not respond to methionine precursors (Murray, I960). When the gas phase was $70 \%(\mathrm{v} / \mathrm{v})$ air $+30 \%(\mathrm{v} / \mathrm{v}) \mathrm{CO}_{2}$ the mutant did not grow on methioninesupplemented medium. Auxanographic experiments showed that the mutant grew on methionine-supplemented medium, in the presence of $30 \%(\mathrm{v} / \mathrm{v}) \mathrm{CO}_{2}$, when adenine or other purines were supplied. Purines therefore reversed the inhibitory effect of $\mathrm{CO}_{2}$. The me-6 mutant 35809 thus seemed to have two defects, one expressed as a requirement for methionine and the other expressed as a requirement for adenine in the presence of $30 \%(\mathrm{v} / \mathrm{v}) \mathrm{CO}_{2}$. It is difficult to understand how such defects could be caused by a single gene mutation, and it seemed that the mutant had mutations in two different genes. However, the requirement for methionine and the sensitivity to inhibition by $\mathrm{CO}_{2}$ did not segregate when the mutant was crossed to wild-type, showing that $\mathrm{CO}_{2}$ sensitivity was caused either by the same mutation as that which caused the methionine requirement (me-6) or by a second mutation closely linked to the me-6 locus. On the assumption that $\mathrm{CO}_{2}$ sensitivity was caused by a second mutation closely linked to $m e-6$, attempts were made to separate it from me-6 by crossing the mutant to mutants lys-3 (28815) and mac (65108), which carry mutations close to, and on either side of, the me-6 locus. Prototrophic segregants arising by recombination in the chromosome interval between me-6 and $l y s-3$ or mac were selected from the crosses and tested for sensitivity to $\mathrm{CO}_{2}$ inhibition. None of the prototrophic segregants obtained was $\mathrm{CO}_{2}$ inhibited. It therefore seems that the $\mathrm{CO}_{2}$ sensitivity of mutant 35809 was caused by the me- 6 mutation.

Recently, a second methionine-requiring mutant me (s2706), kindly given to us by Dr Noreen E. Murray, has been found to resemble mutant me-6 (35809) in its nutritional characteristics. Mutant $m e$ (s2706) is leaky but shows a good growth response to methionine; the methionine-dependent growth is inhibited by $\mathrm{CO}_{2}$, and the $\mathrm{CO}_{2}$ inhibition is reversed by adenine and other purines. The occurrence of a second mutant resembling mutant me-6 (35809) provides additional evidence that the methionine requirement and $\mathrm{CO}_{2}$ sensitivity result from a single gene mutation. Methionine mutants representative of the other known me loci, me-I (35599), me-2 (K 44), me-3 (36I04), me-4 (398I6), me-5 (9666), me-7 (4894), me-8 (P 53) and me-9 (C I 24) were not $\mathrm{CO}_{2}$-inhibited.

When growing in $\mathrm{CO}_{2}$, mutant me-6 (35809) partially resembles mutant mac (65108), which is reported to require methionine+adenine+cysteine for growth (Dubes, I953; Perkins, 1959). This similarity in growth requirement together with the apparent close linkage of mac to me-6 (Perkins, 1959) suggested that the mutants might be allelic.

The nutrition of mac was investigated. It was found that mac grew most quickly with methionine+adenine and less quickly with methionine alone. A requirement for 
cysteine was not demonstrable. $\mathrm{CO}_{2}$ did not inhibit the growth of mac under any conditions. Thus mac differed from me-6 (35809) in that it required adenine for growth under all conditions. Experiments by Dr N. E. Murray (personal communication) have shown that mac (65108), me-6 (35809) and me (s2706) may be allelic.

Of the $\mathrm{CO}_{2}$-inhibited mutants isolated from Escherichia coli only one has been studied. It grew prototrophically in minimal medium in air but its growth was completely inhibited in a gas phase of air $+20 \%(\mathrm{v} / \mathrm{v}) \mathrm{CO}_{2}$. The inhibition by $\mathrm{CO}_{2}$ was reversed by vitamin $\mathrm{B}_{12}$, $\mathrm{L}$ - and $\mathrm{D}$-methionine and was partially reversed by cysteine, cystine, thymidylic acid and DL-serine.

The $\mathrm{CO}_{2}$-inhibited mutant of Salmonella typhimurium similarly grew prototrophically in minimal medium in air and failed to grow in air $+20 \%(\mathrm{v} / \mathrm{v}) \mathrm{CO}_{2}$. The $\mathrm{CO}_{2}$ inhibition of this mutant was specifically reversed by lysine and by the lysine precursor, diaminopimelic acid.

The mutations responsible for the $\mathrm{CO}_{2}$ sensitivity of the bacterial mutants have not been mapped.

There is evidence that $\mathrm{CO}_{2}$ does not inhibit growth of the Neurospora crassa and bacterial mutants by changing the $\mathrm{pH}$ of the medium. Under conditions similar to those of the present experiments, $\mathrm{CO}_{2}$ caused only trivial changes in the $\mathrm{pH}$ of the medium (Broadbent, 1965). Furthermore, the $\mathrm{CO}_{2}$-inhibited mutants are not more sensitive than the parental strains to changes in this $\mathrm{pH}$. It is therefore unlikely that $\mathrm{CO}_{2}$ inhibits growth of the mutants by changing the $\mathrm{pH}$ of the medium. It is possible that un-ionized $\mathrm{CO}_{2}$ may penetrate the cells and change the intracellular $\mathrm{pH}$ after ionization, but this should cause non-specific inhibition and should not be reversed by single metabolites.

\section{DISCUSSION}

Sensitivity to inhibition by $\mathrm{CO}_{2}$ is caused by single gene mutations at least in Neurospora crassa. The $\mathrm{CO}_{2}$ inhibitions are reversed by single metabolites, and the inhibition of each mutant is reversed by a different metabolite or group of related metabolites. These facts show that the $\mathrm{CO}_{2}$ inhibitions are specific and affect particular metabolic processes.

Identification of an impurity in one sample of cytidine which reversed the $\mathrm{CO}_{2}$ inhibition of one of the Neurospora crassa mutants (F4) was unfortunately not possible. It may have indicated the nature of the defect in the mutant and have revealed a new growth factor. This is the second unidentified growth factor encountered in the study of mutants showing $\mathrm{CO}_{2}$ effects; a $\mathrm{CO}_{2}$-requiring mutant of Escherichia coli responds to an unidentified factor in yeast extract (Charles \& Roberts, 1968).

The fact that inosine, adenine and other purines reverse the $\mathrm{CO}_{2}$ inhibition of the methionine-requiring mutant of Neurospora crassa $\left(\right.$ me-6, 35809) suggests that $\mathrm{CO}_{2}$ specifically inhibits an enzyme required for purine biosynthesis. However, the mutation which causes the $\mathrm{CO}_{2}$ sensitivity does not map at a purine locus; both the methionine requirement and the $\mathrm{CO}_{2}$ sensitivity are caused by mutation at the me- 6 locus. This suggests that the me- 6 locus is concerned with the biosynthesis of a substance required for both methionine and purine biosynthesis. Derivatives of folic acid are required for methionine and purine biosynthesis and it is possible that a reaction in their biosynthesis may be controlled by the me-6 locus. Mutant me-6 (35809) may have an incomplete block at this locus, resulting in a requirement for 
methionine only, whereas mutant mac may have a complete block causing a requirement for both methionine and a purine. $\mathrm{CO}_{2}$ may cause the purine requirement of me-6 (35809) by converting the incomplete block into a complete block. Another possible function for the me-6 locus is control of the biosynthesis of S-adenosyl methionine from its precursors, methionine and adenosine triphosphate. Neither of these suggestions explains why mutation at the me-6 locus should cause sensitivity to inhibition by $\mathrm{CO}_{2}$.

The $\mathrm{CO}_{2}$-inhibited mutant of Escherichia coli resembles certain mutants of Salmonella typhimurium which also require methionine or vitamin $\mathrm{B}_{12}$ for growth. It differs from them in that its requirement is expressed only in the presence of a high $\mathrm{CO}_{2}$ concentration. The Salmonella mutants have defects in the enzyme $N^{5}$-methyltetrahydropteroyl-triglutamate-homocysteine transmethylase (Smith \& Childs, I966) which catalyses the direct methylation of homocysteine in the biosynthesis of methionine (Guest, Friedman \& Foster, 1962). Vitamin $\mathrm{B}_{12}$ supports the growth of the Salmonella mutants by stimulating the synthesis of methionine from homocysteine by an indirect mechanism (Guest et al. 1962; Cauthen \& Foster, 1966). It may be that the $E$. coli mutant is unable to effect the direct methylation of homocysteine when the gas phase is air supplemented with $\mathrm{CO}_{2}$, and thereby becomes dependent upon methionine or vitamin $\mathbf{B}_{12}$.

The $\mathrm{CO}_{2}$-inhibited mutant of Escherichia coli also shows similarities with spontaneously occurring variants of Mycobacterium tuberculosis described by Schaefer (1957). Schaefer's variants were inhibited by $\mathrm{CO}_{2}$ when grown on an oleic acid + albumin medium containing glutamic acid as sole organic nitrogen source. This $\mathrm{CO}_{2}$ inhibition was reversed by vitamin $\mathrm{B}_{12}$, L- and D-methionine, DL-homoserine, D-serine and $\mathrm{D}$-alanine and 'less regularly' by various other amino acids.

We can propose no explanation of the apparent inhibition of lysine biosynthesis by $\mathrm{CO}_{2}$ in the $\mathrm{CO}_{2}$-inhibited mutant of Salmonella typhimurium.

The general significance of inhibition by $\mathrm{CO}_{2}$ is not obvious. Species of microorganisms differ widely in their relative sensitivity to $\mathrm{CO}_{2}$. It may be that $\mathrm{CO}_{2}$ inhibits some micro-organisms by causing non-specific effects such as $\mathrm{pH}$ shifts, but it is more difficult to explain large differences in $\mathrm{CO}_{2}$ sensitivity in the same way. The occurrence of $\mathrm{CO}_{2}$-sensitive mutants raises the possibility that inhibition of wild-type strains by $\mathrm{CO}_{2}$ may often be due to effects on single enzymes.

The $\mathrm{CO}_{2}$ effects shown by the $\mathrm{CO}_{2}$-inhibited and $\mathrm{CO}_{2}$-stimulated mutants of Neurospora crassa, Escherichia coli and Salmonella typhimurium suggest that the role of $\mathrm{CO}_{2}$ in cell physiology is more complex than might be expected from our present knowledge of $\mathrm{CO}_{2}$ metabolism.

The authors wish to acknowledge a Science Research Council Studentship awarded to G.A.R., a research grant from the Agricultural Research Council and the technical asisstance of Mr Ian McMurray. 


\section{REFERENCES}

Beadle, G. W. \& Tatum, E. L. (1945). Methods of producing and detecting mutations concerned with nutritional requirements. American Journal of Botany 32, 678-686.

Broadbent, J. A. (1965). Carbon dioxide-requiring Mutants in Neurospora crassa. Ph.D. Thesis, University of Reading.

Broadbent, J. A. \& Charles, H. P. (1965). Some carbon dioxide-requiring mutants of Neurospora crassa. Journal of General Microbiology 39, 63-74.

CAtCheside, D. G. (I954). Isolation of nutritional mutants of Neurospora crassa by filtration enrichment. Journal of General Microbiology II, 34-36.

Cauthen, G. E. \& Foster, M. A. (1966). Personal communication cited in Smith, D. A. \& Childs, J. D. (I966). Methionine genes and enzymes of Salmonella typhimurium. Heredity 21, 265-286.

Charles, H. P. (I964). Relationships between certain pyrimidine and arginine mutants of Neurospora as revealed by their response to $\mathrm{CO}_{2}$. Journal of General Microbiology 34, I3I-142.

Charles, H. P. \& Broadbent, J. A. (1964). Carbon dioxide mutants: a large and interesting class of Neurospora mutants. Nature, London 201, $1004-1006$.

Charles, H. P. \& Roberts, G. A. (I968). Carbon dioxide as a growth factor for mutants of Escherichia coli. Journal of General Microbiology 5I, 21 I-224.

DuBEs, G. R. (I953). Investigations of some 'unknown' mutants of Neurospora crassa. Ph.D. Thesis, California Institute of Technology.

Guest, J. R., Friedman, S. \& Foster, M. A. (I962). Alternative pathways for the methylation of homocysteine by Escherichia coli. Biochemical Journal 84, 93 P.

Lederberg, J. \& Lederberg, E. M. (1952). Replica plating and indirect selection of bacterial mutants. Journal of Bacteriology 63, 399-406.

Murray, N. E. (1960). The distribution of methionine loci in Neurospora crassa. Heredity $\mathbf{1 5}_{\mathbf{5}}$, 199206.

Perkins, D. D. (I959). New markers and multiple point linkage data in Neurospora. Genetics 44, I I 85I 208.

Pontecorvo, G. (1949). Auxanographic techniques in biochemical genetics. Journal of General Microbiology 3, 122-1 26.

RYAN, F. J. (1950). Selected methods of Neurospora genetics. Methods in Medical Research 3, 5I -75.

SCHAEFER, W. B. (1957). Studies on the inhibiting effect of carbon dioxide on the growth of two mutant strains of Mycobacterium tuberculosis. Journal of Bacteriology 73, 52-55.

Smith, D. A. \& Childs, J. D. (I966). Methionine genes and enzymes of Salmonella typhimurium. Heredity 21, 265-286.

Sмiтн, G. (1954). An Introduction to Industrial Microbiology, 4th edn, p. 239. London: Edward Arnold.

Vogel, H. J. (1956). A convenient growth medium for Neurospora (Medium N). Microbial Genetics Bulletin $\mathbf{1 3}, 42-43$.

Vogel, H. J. \& BonNer, D. H. (I956). A convenient growth medium for $E$. coli and some other micro-organisms (Medium E). Microbial Genetics Bulletin 13, 43-44.

Woodward, V. W., De Zeeuw, J. R. \& SRB, A. M. (1954). The separation and isolation of particular biochemical mutants of Neurospora by differential germination of conidia, followed by filtration and selective plating. Proceedings of the National Academy of Sciences of the United States of America 40, 192-200. 